\title{
Recent advances in nanoparticle carriers for photodynamic therapy
}

\author{
Gawon Yi ${ }^{1}$, Suk Ho Hong ${ }^{2}$, Jihwan Son ${ }^{1}$, Jihye Yoo ${ }^{1}$, Changhee Park ${ }^{1}$, Yongdoo Choi ${ }^{2}$, Heebeom Koo ${ }^{1,3}$ \\ ${ }^{1}$ Department of Medical Lifescience, College of Medicine, The Catholic University of Korea, Seoul, Republic of Korea; ${ }^{2}$ Biomarker Branch, \\ National Cancer Center, Goyang-si, Gyeonggi-do, Republic of Korea; ${ }^{3}$ Catholic Photomedicine Research Institute, College of Medicine, The \\ Catholic University of Korea, Seoul, Republic of Korea
}

Correspondence to: Heebeom Koo. Department of Medical Lifescience, College of Medicine, The Catholic University of Korea, 222 Banpo-daero, Seoul 06591, Republic of Korea. Email: hbkoo@catholic.ac.kr.

\begin{abstract}
This review summarizes recent advances in the development of nanoparticles (NPs) for efficient photodynamic therapy (PDT), particularly the development and application of various NPs based on organic and inorganic materials. PubMed database was used for literature search with the terms including NP, nanomedicine, PDT, photosensitizer (PSs), and drug delivery. For successful PDT, it is essential to deliver PSs to target disease sites. A number of NPs have been developed and tested as the carriers for both imaging and therapy, an approach termed "nanomedicine". Many studies of NP carriers showed increased water solubility and stability of PSs for in vivo injection, and these NP carriers provided benefits including longer circulation in blood and higher accumulation of PSs at disease sites. This review describes new techniques in PDT such as aggregation-induced emission (AIE) and luminescence-based PDT, and provides insights on NPs and PDT for biomedical researchers working to develop or apply NPs in efficient PDT.
\end{abstract}

Keywords: Photodynamic therapy (PDT); nanomedicine; nanoparticle (NP); imaging; drug delivery

Submitted Apr 24, 2018. Accepted for publication May 12, 2018.

doi: 10.21037/qims.2018.05.04

View this article at: http://dx.doi.org/10.21037/qims.2018.05.04

\section{Introduction}

Photodynamic therapy (PDT) is a clinical treatment based on the activation of light-absorbing molecules, or photosensitizers (PSs). Upon light irradiation at a specific wavelength, PSs generate reactive oxygen species (ROS), which are toxic to the targeted disease cells. PSs are initially in a singlet state at the ground state energy level $\left(\mathrm{S}_{0}\right)$ and they change to the excited state $\left(\mathrm{S}_{1}\right)$ after absorbing photons of light (1). Because this excited state is unstable and short-lived, PSs return to their ground state by providing fluorescence or transferring to the excited triplet state $\left(\mathrm{T}_{1}\right)$, which is relatively more stable than $S_{1}$. Then, the PSs at $\mathrm{T}_{1}$ can transfer hydrogen, electron, or direct energy to the surrounding oxygen and generate ROS. Typically, nearinfrared (NIR) lasers within 600- to 800-nm wavelength have been used for PDT to prevent interference by endogenous chromophores in tissues (2). Currently, PDT is being widely tested or administered in clinics for cancer therapy of the skin, prostate, head and neck, pancreas, breast, and lung (3).

A representative advantage of PDT is that there is no cross-resistance between PDT and chemotherapy, because its mechanism is different from traditional chemotherapy (4). Consequently, PDT can be effective against multi-drugresistant cancer cells, which have recently been regarded as the main reason for the incomplete and failed results of chemotherapy (5). This makes PDT a promising synergistic or an alternative treatment to chemotherapy. In addition, even though the need for laser light activation limits the application of PDT, this can still be an advantage in terms of side effects: it means that the toxicity of PSs is much lower in the unirradiated regions, which is also different from most drugs. 
As with chemotherapy, an efficient drug delivery system is one of the major challenges in PDT. Because most PSs are hydrophobic with poor water solubility, many researchers have sought to identify methods to encapsulate and solubilize PSs in aqueous conditions for the injection into the human body (6). Ideally, the delivery system should be biocompatible, able to prevent aggregation, and provide high accumulation of PSs in the target disease site with minimized uptake by normal cells. In recent years, various nanoparticles (NPs) have been developed for the use as drug carriers or imaging probes due to the following advantages $(7,8)$. First, they can increase the solubility of hydrophobic drugs by physical loading and chemical conjugation in aqueous conditions. Second, their size is suitable for intravenous injection by syringe and providing longer blood circulation than that achieved by small molecules. In particular, macromolecules such as polymers or NPs can pass through the gaps in the walls of newly-grown vessels in angiogenic diseases like tumors. Furthermore, they are secreted slowly due to the reduced lymphatic drainage, and this situation has been called the enhanced permeation and retention (EPR) effect (9). Third, the release of drugs can be controlled by changing the structure of NPs. Fourth, the surfaces of NPs can be decorated with biological ligands including antibodies, aptamers, or peptides, which can bind to specific receptors on target cells. It results in the increased specificity $(10,11)$. Fifth, both imaging agents and drugs can be contained in a single NP, which enables simultaneous imaging and therapy, an approach termed as theranostics (12). These advantages have made NPs promising carriers for PSs in PDT, and a large number of papers have reported positive results $(13,14)$. Therefore, in this review, we will introduce the recently-developed NPs that are useful for PDT. We will describe traditional organic NPs such as polymeric NPs or liposomes and inorganic NPs including quantum dot (QD), silica, and upconversion NP materials. In particular, new techniques in PDT such as aggregation-induced emission (AIE) and luminescencebased PDT will be introduced.

\section{NPs for PDT}

\section{Traditional physical loading or chemical conjugation of $P S$ s in organic materials}

Traditional organic NPs such as liposomes or polymeric micelles have been studied as drug carriers. Generally, they are fabricated based on the self-assembly of components, so that they have amphiphilic structures in water. This means that they have hydrophobic space inside, which can accommodate hydrophobic drugs like PSs. Phospholipids or polymers including FDA-approved synthetic polymers or biopolymers are popular materials for the creation of organic NPs. Because the amphiphilic structure can be dissociated after cellular uptake in general, these kinds of NPs return to their components after a period of time in the body. This situation is advantageous for both drug release and minimizing the toxicity of NPs.

In 2018, Son et al. used folate-modified poly(lactic-coglycolic acid) (PLGA) NPs for the tumor-targeted delivery of PSs (15). The PLGA cores of the NPs were suitable for loading hydrophobic PSs. On the surfaces of many cancer cells, folate receptors are overexpressed. To target them, the surfaces of the NPs consisted of phospholipids modified with polyethylene glycol (PEG) and folate. Hydrophobic pheophorbide a $(\mathrm{Pba})$ was used as a PS and was loaded onto the NPs for PDT with over $80 \%$ loading efficiency. These folate-PLGA-Pba NPs enhanced the cellular uptake and killing effect in MKN28 human gastric cancer cells with overexpressed folate receptors. In vivo imaging based on the intrinsic fluorescence of $\mathrm{Pba}$ showed a high accumulation of these NPs at the tumor site in the MKN28 tumor-bearing mouse model.

A similar encapsulation of PSs into amphiphilic polymer NPs was also performed by Yoon et al. (16) (see Figure 1). They used hyaluronic acid (HA) NPs (HANPs) to carry a hydrophobic PS, chlorin e6 (Ce6). The chemical conjugation of aminated $5 \beta$-cholanic acid, PEG, and black hole quencher3 (BHQ3) to the HA backbone resulted in self-assembled HANPs. Ce6 was loaded into the HANPs by a simple dialysis method under aqueous conditions. The strategy for targeting the tumor site with the HANPs was based on the binding of HA to CD44 receptors as well as the EPR effect. Ce6-loaded-HANPs rapidly released Ce6 in the tumor tissue due to the degradation of the HA backbone in presence of the hyaluronidase abundant in the cytosol of tumor cells. The role of BHQ3 was to quench the fluorescence and PDT effect of Ce6 before the release of Ce6 from the NPs. After 30 min of incubation with Ce6HANPs, the researchers observed fast cellular uptake and intracellular fluorescence generation of Ce6-HANPs in HT29 cells, and the fluorescence intensity was higher than in the NIH3T3 fibroblast cells used as a control. HT29 cells treated with Ce6-HANPs were damaged after 2 min of irradiation with an NIR laser, and their viability was lower than $10 \%$. After intravenous injection of Ce6-HANPs into 

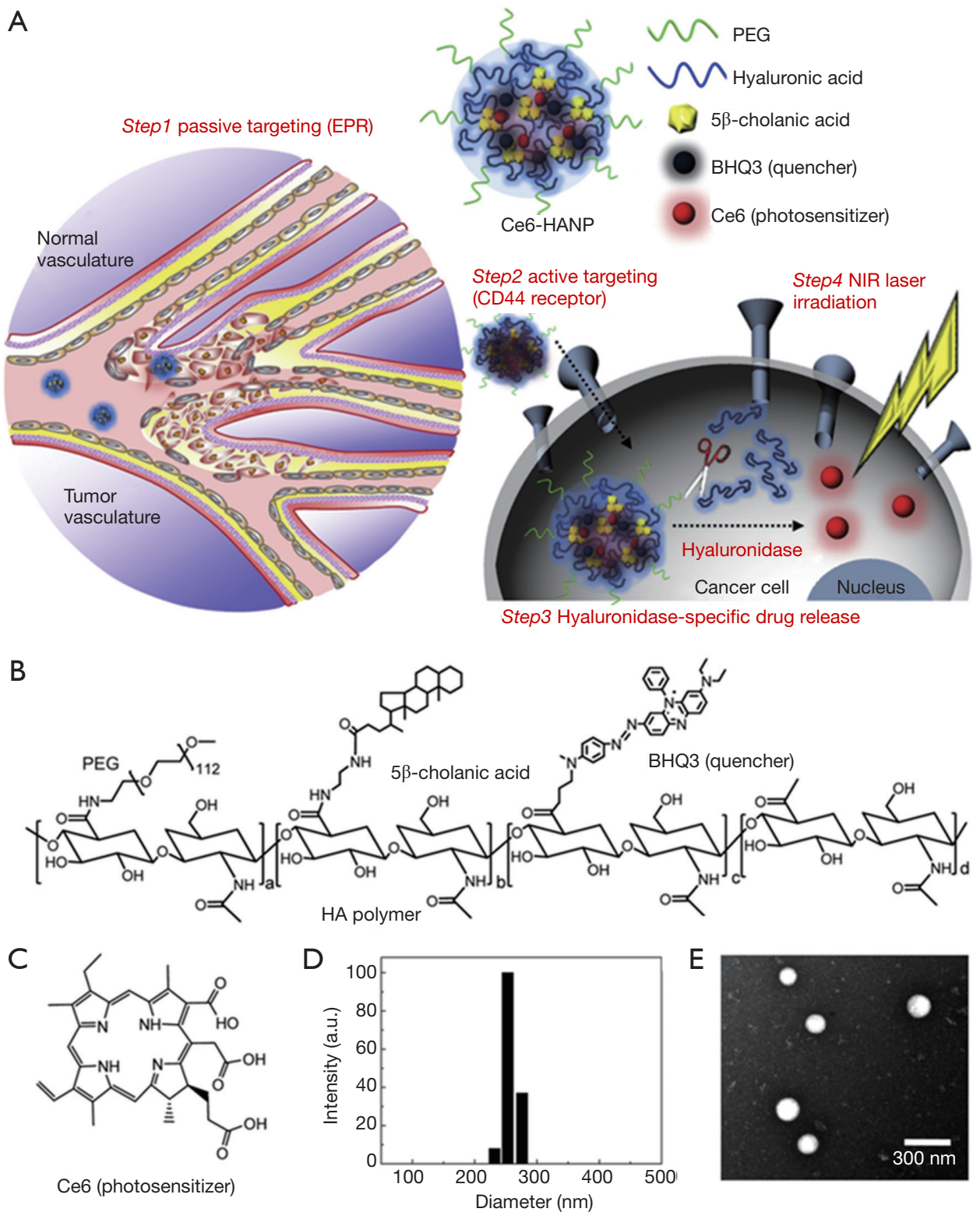

Figure 1 Chlorin e6-loaded hyaluronic acid nanoparticles for photodynamic therapy (PDT). (A) Illustration of tumor-targeting strategy; (B) chemical structure of the nanoparticle; (C) chemical structure of chlorin e6; (D) size distribution; (E) transmission electron microscopy image. Reproduced with permission from (16). PEG, polyethylene glycol.

tumor-bearing mice, the NPs were effectively accumulated at the tumor sites, and ex vivo analysis also showed that the tumor tissue had the strongest fluorescence signal among the organs. Upon laser irradiation, the accumulated Ce6HANPs in tumor tissue provided high therapeutic efficacy when the tumor growth rate was compared to that of the control groups.

In contrast to other chemical drugs, PSs can provide a therapeutic effect while they are conjugated to other materials like polymers or NPs, because the generated ROS kill the cells, not the PSs themselves. A recent study by Luo et al. is a good example of NPs based on conjugation between polymers and PSs (17). They conjugated pyropheophorbide a as a PS to poly(vinyl alcohol) (PVA) by ester bond to make an amphiphilic structure, which formed a self-assembled micelle structure. Then, hydrophobic 
doxorubicin (DOX) was loaded into the core of NPs via $\pi-\pi$ stacking between DOX and pyropheophorbide a. In addition, ${ }^{64} \mathrm{Cu}^{2+}$ was chelated into the porphyrin structure of pyropheophorbide a. The resulting NPs were intravenously injected into the tumor-bearing mice, and transferred to the tumor by the EPR effect. After irradiation by a $690-\mathrm{nm}$ laser $\left(0.5 \mathrm{~W} / \mathrm{cm}^{2}\right.$ for $\left.2 \mathrm{~min}\right)$ of the tumor 24 hours postinjection, the survival rate increased up to $80 \%$ by day 40 , and the tumor volume was markedly decreased by PDT compared to those of other groups. Furthermore, co-loaded DOX and ${ }^{64} \mathrm{Cu}^{2+}$ can also be used for chemotherapy and positron emission tomography, respectively.

In 2016, Spring et al. introduced rationally designed liposomes for combination therapy (18). They developed photoactivatable multi-inhibitor nanoliposomes (PMILs) for the photoinitiated and sustained release of inhibitors as well as PDT. The PMILs contained a photoactivable chromophore (benzoporphyrin derivative, BPD) as PS in the lipid bilayer, and also encapsulated smaller PLGA NPs loading cabozantinib (XL184), a multikinase inhibitor. NIR light could activate PMILs by disrupting the bilayer of liposomes and releasing XL184, which could inhibit the anti-apoptotic signaling pathways that help cell survival. The sustained release of XL184 inhibited vascular endothelial growth factor (VEGF) signaling in the tumor microvasculature to suppress tumor angiogenetic activity and vascular regrowth after PDT. Also, XL184 inhibited MET signaling with the suppression of tumor cell motility, invasion, and metastasis. The effective inhibition of both VEGF and MET signaling was confirmed during in vitro and in vivo studies. PMIL treatment showed a reduction in tumor volume in an AsPC1 tumor xenograft mice model based on PDT and photoinitiated drug release. Furthermore, PMIL treatment exhibited a reduction of liver and retroperitoneal lymph node metastasis and also decreased intratumoral microvessel volume in a pancreatic ductal adenocarcinoma tumor model.

For successful combination therapy, the selection of a drug synergetic with PDT is also important. Recently, Wang et al. developed iRGD-modified NPs for the simultaneous tumor delivery of indocyanine green (ICG) and a hypoxia-activated prodrug, tirapazamine (TPZ) (19). PDT consumes oxygen for therapy and induces hypoxia upon laser irradiation. Therefore, Wang et al. hypothesized that the toxicity of TPZ could increase when it was delivered with PSs and localized in a hypoxic environment after PDT. They simultaneously added TPZ and ICG into dibenzocyclooctyl-modified PEG-PLGA NPs. Next, azido- functionalized iRGD peptide was conjugated to surface of NPs by click chemistry for increased vascular/tissue permeability and cellular uptake. The in vivo results in orthotopic breast tumor models showed that ICG induced hypoxia as well as PDT did and increased the anticancer activity of simultaneously delivered TPZ.

\section{Combination with inorganic materials}

Recent advances in inorganic chemistry and their application to nanotechnology have resulted in various inorganic NPs, which have contributed greatly to NP research. The reason that inorganic NPs have attracted much attention is that each inorganic material has its intrinsic functions that are useful for biomedical applications. For example, iron oxide NPs can be used as a T2 magnetic resonance imaging contrast agent, and gold NPs have a photothermal effect due to their surface plasmon resonance. However, the injection of inorganic materials into the human body is thought to be riskier than the injection of traditional organic materials, because most of the inorganic materials did not originally exist in the body. Therefore, during the development of NPs with inorganic materials, the evaluation of acute and potential toxicity needs to be considered carefully.

Mesoporous silica NP is a promising material with a large surface area, a high capacity to load cargo in pores, and facile size control. In 2017, Wang et al. developed a novel method to synthesize self-assembled porphyrincontaining mesoporous silica NPs (see Figure 2) (20). The synthesized NPs were composed of zinc-tetra (4-pyridyl) porphyrin (ZnTPP) as a PS, amorphous silica shells, and surfactant. Two different porphyrin-silica NPs, including core-shell particles $\left(\mathrm{ZnTPP} @ \mathrm{SiO}_{2}\right)$ and solid particles $\left(\mathrm{ZnTPP}-\mathrm{SiO}_{2}\right)$, were synthesized by precise control of the non-covalent interactions of ZnTPP within surfactant micelles. Under acidic conditions, ZnTPP promotes selfassembly surrounded later with a silica shell, while at high $\mathrm{pH}$, porphyrin self-assembly is comparatively slow and forms solid NPs. Both types of silica NPs could generate ROS to kill cancer cells by PDT. They also exhibit intense fluorescence for cell labeling, and the silica does not cause toxicity after cellular uptake. When they were conjugated with folate for active targeting, both types of silica NPs could destroy HeLa cells more effectively in vitro. In addition, the PDT effect was stronger in the case of the core-shell-type NPs due to their higher ROS generation efficiency.

Upconversion fluorescent NPs (UCNPs) are generally 


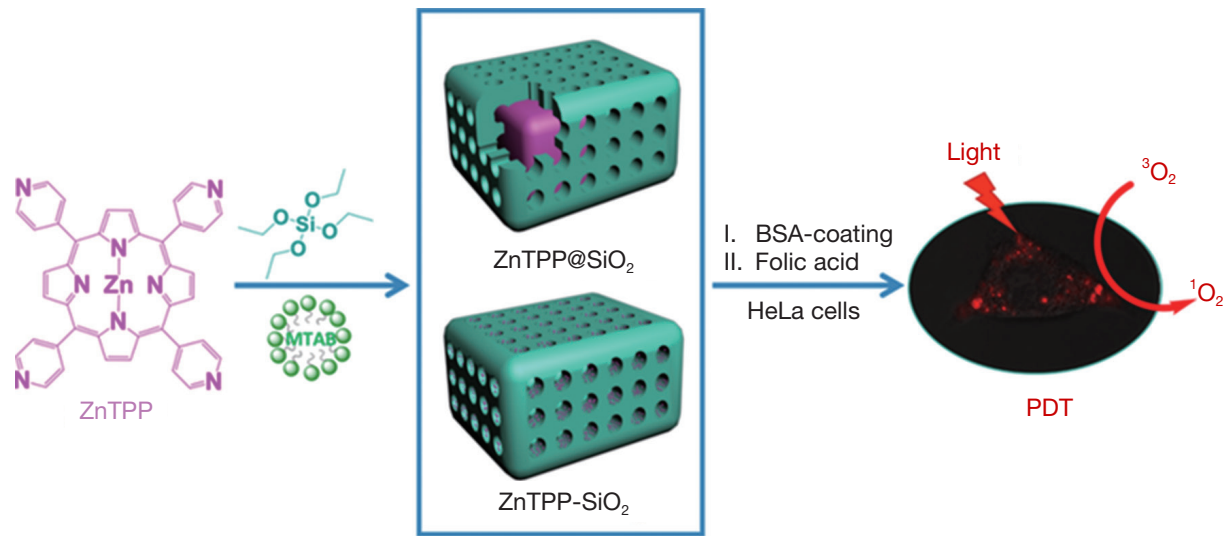

Figure 2 Porphyrin-containing mesoporous silica nanoparticles for PDT. Reproduced with permission from (20). PDT, photodynamic therapy.

made from rare-earth metals, and can absorb and convert NIR light into visible light that could activate PS. This strategy has great advantages to penetrate deep tissues that visible light cannot reach. Idris et al. developed a new type of UCNPs as a nano-transducer to overcome the conventional limitations of PDT (21). UCNPs had a controllable size of about $100 \mathrm{~nm}$, and the coated mesoporous silica shell layers were suitable for encapsulating two kinds of PSs, $\mathrm{ZnPc}$ and MC540. They also utilized the multicolor emission capability of the UCNPs, which could activate the two PSs simultaneously using a single excitation wavelength. The resulting UCNPs showed an enhanced singlet oxygen generation rate and cancer cell death, providing greater efficiency of PDT than in the case using a single PS. During in vivo studies, this combination of dual PSs and UCNPs effectively activated at a single $980-\mathrm{nm}$ wavelength laser irradiation and reduced tumor growth. In addition, when they used folate-modified NPs, the targeting ability and therapeutic effect were further enhanced in a B16F10 melanoma tumor-bearing mouse model.

Recently, carbon nanomaterials such as nanotubes and graphene have also attracted a great deal of attention due to their special optical properties, including strong absorbance and photothermal effects. An interesting study by Ge et al. showed a rational design of graphene for PDT (22). They synthesized graphene quantum dot (GQDs) with polythiophene derivatives with effective singlet oxygen generation based on multistate sensitization mechanism. GQDs dispersed well in water and showed a broad absorption band in the UV-visible region and a strong far-red emission $(680 \mathrm{~nm})$. Specifically, GQDs provided a high quantum yield of singlet oxygen and good biocompatibility. GQDs could destroy more cancer cells than protoporphyrin IX (PpIX), a conventional PS, in low concentrations in vitro. In addition, GQDs could facilitate in vivo imaging simultaneously with PDT based on their intense fluorescence. GQDs could inhibit tumor growth by PDT due to their high singlet oxygen quantum yield without weight change in a tumor-bearing mouse model.

\section{AIE}

Common wisdom dictates that fluorophores are fluorescent in dilute solutions, but once the molecules are aggregated, their fluorescence is decreased or quenched altogether. This phenomenon, called aggregation-caused quenching, has been studied thoroughly (23), and its mechanism was also elucidated: aromatic rings, which most fluorophores contain, are subject to strong intermolecular $\pi-\pi$ stacking interaction, and once aggregates are formed, excited states are relaxed via non-radiative routes. This property has been utilized in the field of PDT in many studies $(24,25)$. However, other special types of molecules attain the opposite of this, resulting in what is called AIE. Here, the luminogens (called AIEgens) are non-emissive as molecules, but when aggregates are formed, they become emissive (23). For example, an archetypical AIEgen is tetraphenylethene (TPE), with four phenyl rings freely connected to a central olefin structure. In solution state, the rotation of these rings provides a non-radiative way of dissipating exciton energy. As a result, TPE has almost no fluorescence in dilute solution phase. However, when TPE is aggregated by, for example, the introduction of a poor solvent such as water, rotation is physically restrained (referred to as the restriction 

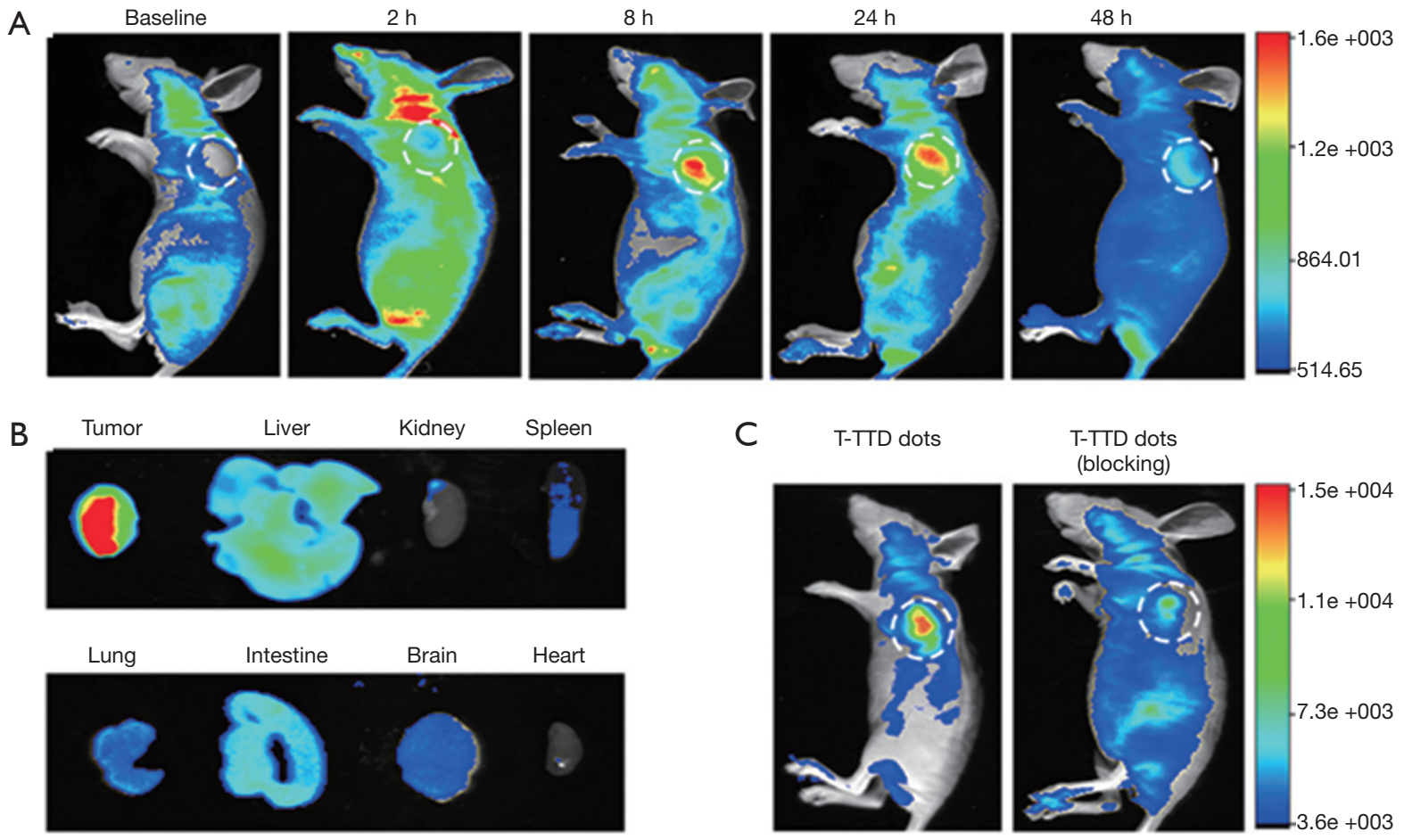

Figure 3 Image-guided PDT with targeted aggregation-induced emission (AIE) dots. (A) Biodistribution of an AIE theranostic probe (denoted as T-TTD dots) injected intravenously, as seen in fluorescence imaging; (B) ex vivo images of organs and tumor tissue from injected mice; (C) images with and without blocking the integrin $\alpha_{v} \beta_{3}$, to demonstrate the targeting abilities of T-TTD dots. Reproduced with permission from (27).

of intramolecular motion) and, moreover, the twisted structure of this molecule hinders $\pi-\pi$ stacking interaction. This process leads to the emergence of fluorescence (23).

This property creates interesting opportunities for PDT as well, and several studies have applied AIE for PDT, some of which we will discuss in this section. Mainly, AIE can be used to create $\mathrm{NP}_{\mathrm{S}}$ - or dots-with bright fluorescence, due to the aggregation of fluorophores inside the NPs; also, it can be used to create some sort of responses to the environment. In the former, AIE enables the imaging and subsequent localization of therapy (image-guided therapy) by the user. Since 2014, when a molecule with two triphenylamine (TPA), an AIEgen, and two TPE moieties and a red-emitting 2-(4H-pyran-4-ylidene)malononitrile part in the center (TTD) was used to create dots, which were then encapsulated in amphiphilic PEG conjugates (26), many works were done in this fashion. For example, Li et al. attached cRGD to the end of the amphiphilic PEG, targeting integrin $\alpha_{v} \beta_{3}$ overexpressing cholangiocarcinoma cells (27) (see Figure 3). In another study, TPE conjugated to dicyanovinyl was used, and it showed a high twophoton absorption cross section and promising two-photon imaging capability (28). Jayaram et al. conjugated TPE to benzothiazole derivatives that self-assembled into NPs and demonstrated its efficacy on PC3 human prostate cancer cells, both in vitro and in vivo (29).

In other researches, the probe was made to be responsive to the surrounding environment. Sometimes organelle targeting functionality is used, because it brings the AIEgens together intracellularly. In 2012, 3,6-bis-(1-methyl-4vinylpyridinium)-carbazole diiodide (BMVC) was used as a AIEgen that passes the excitation energy onto a porphyrin moiety (30). BMVC was also used to discern cancer cells from normal cells due to its higher uptake by tumor cells and its increase in quantum yield upon contact with DNA. This complex of self-assembled BMVC-porphyrin conjugates was evaluated for its imaging and PDT capabilities in HeLa cells. In 2015, Yuan et al. used a TPE derivative conjugated with a peptide sequence and cRGD to create a cathepsin B-sensitive probe. This was found to 


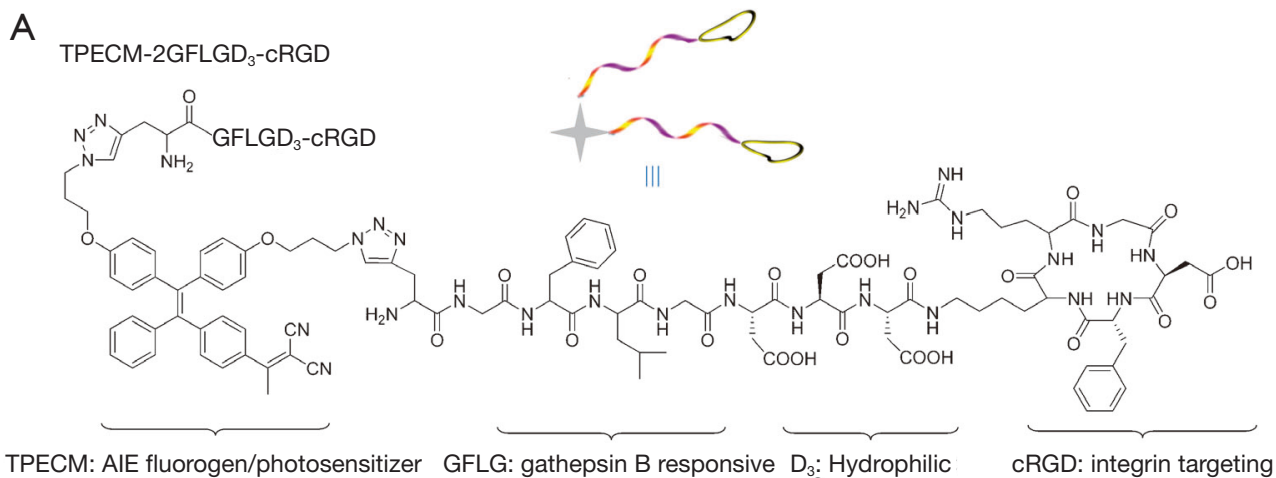

B

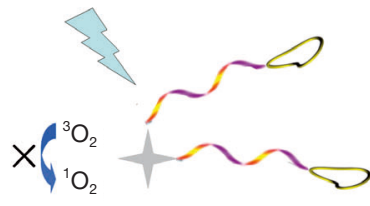

Fluorescence "off"

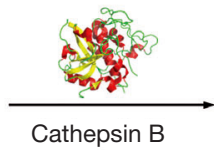

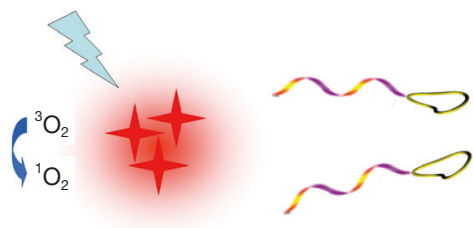

Fluorescence "on"

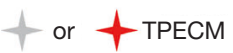

$\sim \mathrm{GFLGD}_{3} \circlearrowright \mathrm{cRGD}$

Figure 4 Enzyme-responsive nanoprobes with AIE for targeted PDT. (A) Breakdown of an AIE probe attached by a responsive linker to a targeting moiety; (B) schematic illustration of how fluorescence and reactive oxygen species generation of this probe turns on upon contact with cathepsin B. Reproduced with permission from (31). AIE, aggregation-induced emission; PDT, photodynamic therapy.

have specific uptake by an integrin-overexpressing cell line (MDA-MB-231) over others (31) (see Figure 4). In 2015, Feng et al. synthesized a TPE derivative that can generate singlet oxygen, DPBA-TPE, created nanosized dots from it and PEG derivatives with a folic acid moiety, and reacted with triphenylphosphine (TPP) for mitochondria targeting purposes (32). The resulting particles, dubbed FA-AIETPP, showed selective phototoxicity against folate receptorpositive MCF-7 breast cancer cells, and proved useful for image-guided PDT. In another study, a complex of TPE, PEG-peptide linker, and a PS (PpIX) was used (33). When the cell contained overexpressed matrix metalloproteinase-2 (MMP-2), the MMP-2 cleaved the peptide linker, and the freed TPE exhibited AIE. Thus, researchers were able to discern solid tumor cells and selectively irradiate them for PDT. Also, UCNPs have been used in conjunction with a polymer designed to exhibit AIE (34). Here, a $\mathrm{pH}$-sensitive linker allowed the PEG layer to be shed once the NPs were in an acidic environment of tumor cells, after which the TPP moiety on the NPs allowed mitochondrial targeting. UCNPs, together with the AIEgen, were shown to generate ROS efficiently under NIR irradiation, which allowed effective PDT against A549 human lung cancer cells.

\section{Luminescence-based PDT}

Despite the advantages of PDT, PDT has an intrinsic limitation in that it can be applied only to the areas where light can be supplied. This limitation has inhibited wider applications of PDT to various diseases that occur in inner organs or tissues. Recently, some researchers have focused on bioluminescence or chemiluminescence to overcome this limitation of traditional PDT. They wanted to generate and use light from particular chemical reactions with small molecules or proteins without excitation by an external light source such as a laser. Ideally, this could enable PDT without light, so that the process is free from the disadvantages of current PDT, the light application and its penetration depth.

In 2012, Yuan et al. activated PSs using chemical molecules without laser irradiation. They used a chemical reaction between luminol, horseradish peroxidase (HRP), and hydrogen peroxide (35). The generated luminescent light of broad wavelength $(400-500 \mathrm{~nm})$ from these molecules was used to activate cationic oligo (p-phenylene vinylene) (OPV), the synthesized PS. The cationic charges of OPV facilitate its binding to the cell surface and uptake. 
When HeLa cells were treated with OPV, HRP, luminol, 4-iodophenol, and $\mathrm{H}_{2} \mathrm{O}_{2}$, their viability decreased in a manner dependent upon the concentration of OPV. Then, the mixture was administered to HeLa cell tumor-bearing mice by intratumoral injection, and tumor growth was observed for 19 days. Only the mice injected with every component including luminol, HRP, and OPV showed tumor suppression, while tumor growth in other groups without OPV or luminol was similar to that of the control group. This study demonstrated that chemiluminescence without external light excitation can be used for PDT, but the usage of a mixture including multiple materials needs to be changed for easier application.

Recently, Mao et al. developed another chemiluminescencebased system for PDT (36). They encapsulated bis[2,4,5trichloro-6-(pentyloxycarbonyl)phenyl] oxalate (CPPO) and their synthesized PS into NPs composed of soybean oil cores and Pluronic F127 shells. CPPO reacts with $\mathrm{H}_{2} \mathrm{O}_{2}$ and generates a 1,2-dioxetanedione intermediate, which can excite a PS. The PS called TBD was synthesized with three molecules, methoxy-substituted TPE, dicyanovinyl, and benzothiadiazole, as the electron donor, electron acceptor, and auxiliary acceptor, respectively. It is a kind of AIE PS, which has been explained above, enabling efficient singlet oxygen generation from aggregated PSs inside NPs. In a $4 \mathrm{~T} 1$ breast cancer tumor model, the NPs showed a significant chemiluminescence signal in tumor tissue because of EPR effect-based delivery and activation by $\mathrm{H}_{2} \mathrm{O}_{2}$ in tumor tissue. To increase the activation of CPPO further, they used beta-phenylethyl isothiocyanate (FEITC), an anticancer drug that can enhance $\mathrm{H}_{2} \mathrm{O}_{2}$ generation in tumors. With the synergistic effect of FEITC, the NPs resulted in a strong photodynamic effect in mouse models and complete suppression of the tumor growth for 14 days.

In addition to chemiluminescence studies, some researchers were interested in bioluminescence with natural proteins like luciferase. In 2013, Hsu et al. (37) introduced luciferase-based self-illuminated PDT without excitation with light. They conjugated Renilla luciferase 8 (RLuc8) onto the surface of QDs. They also used metatetra-hydroxyphenyl-chlorin (mTHPC)-loaded micelles as PSs. When a QD-RLuc8 conjugate met the substrate coelenterazine, bioluminescence was generated. QD was used to transduce the broad absorbance of light into emission with a particular wavelength, which is called bioluminescence resonance energy transfer. The emitted photons can stimulate mTHPC in the micelles and generate ROS for PDT. The size of this QD-RLuc8 conjugate was about $20 \mathrm{~nm}$, and the generated ROS in presence of coelenterazine was observed using the fluorescent dye $2^{\prime}, 7^{\prime}$-dichlorodihydrofluorescein diacetate as an indicator. This system successfully killed A549 tumor cells and induced apoptosis, which was analyzed by TUNEL staining. When the QD-RLuc8 conjugate and mTHPC-loaded micelles were directly injected into tumors and coelenterazine was injected intravenously, tumor growth was effectively delayed by PDT in vivo. In 2015, Yun's group also demonstrated a similar type of bioluminescence-based PDT using RLuc8, QD, and Ce6 as the PS (38). Based on this result, the same group showed an improved system in their next study published in 2017 (39). They directly conjugated RLuc 8.6 with rose bengal, a PS with absorbance at around $550 \mathrm{~nm}$ (see Figure 5). The broad spectrum of bioluminescence from RLuc 8.6 could overlap with the absorption of rose bengal, so that it could generate ROS without a QD transducer. Although this study did not include animal data, these kinds of trials are exciting and will be helpful in overcoming the current limitations of PDT.

\section{Conclusions}

To date, numerous studies have investigated the use of NPs for efficient PDT, and many of them are still ongoing. This trend may be related to the advantages of PDT, including its effectiveness against drug-resistant cells due to its different mechanism and the capacity for controlled therapy triggered with light. In particular, the intrinsic fluorescence of a PS itself as well as its ROS-generating ability is highly useful for both research and applications. For researchers, the concentration of PS in solution can be determined by its light absorbance or fluorescence emission. This makes it easier to obtain the loading efficiency of PS in NPs or the drug release in vitro without HPLC analysis. Furthermore, the biodistribution of PSs in animal models or blood circulation can also be analyzed by their fluorescence. It means that if they are specifically delivered to the target disease site after injection, the NPs can also be used as fluorescence imaging probes for simultaneous diagnostics and therapy or theranostics. These advantages of PSs are highly attractive to researchers, so that many kinds of studies have been performed to deliver PSs to the target disease sites for successful PDT. Various organic or inorganic NPs have been developed and have shown positive results in animal experiments. Specifically, recent techniques including AIE and luminescence-based PDT are promising approaches to the current limitations of PDT. 


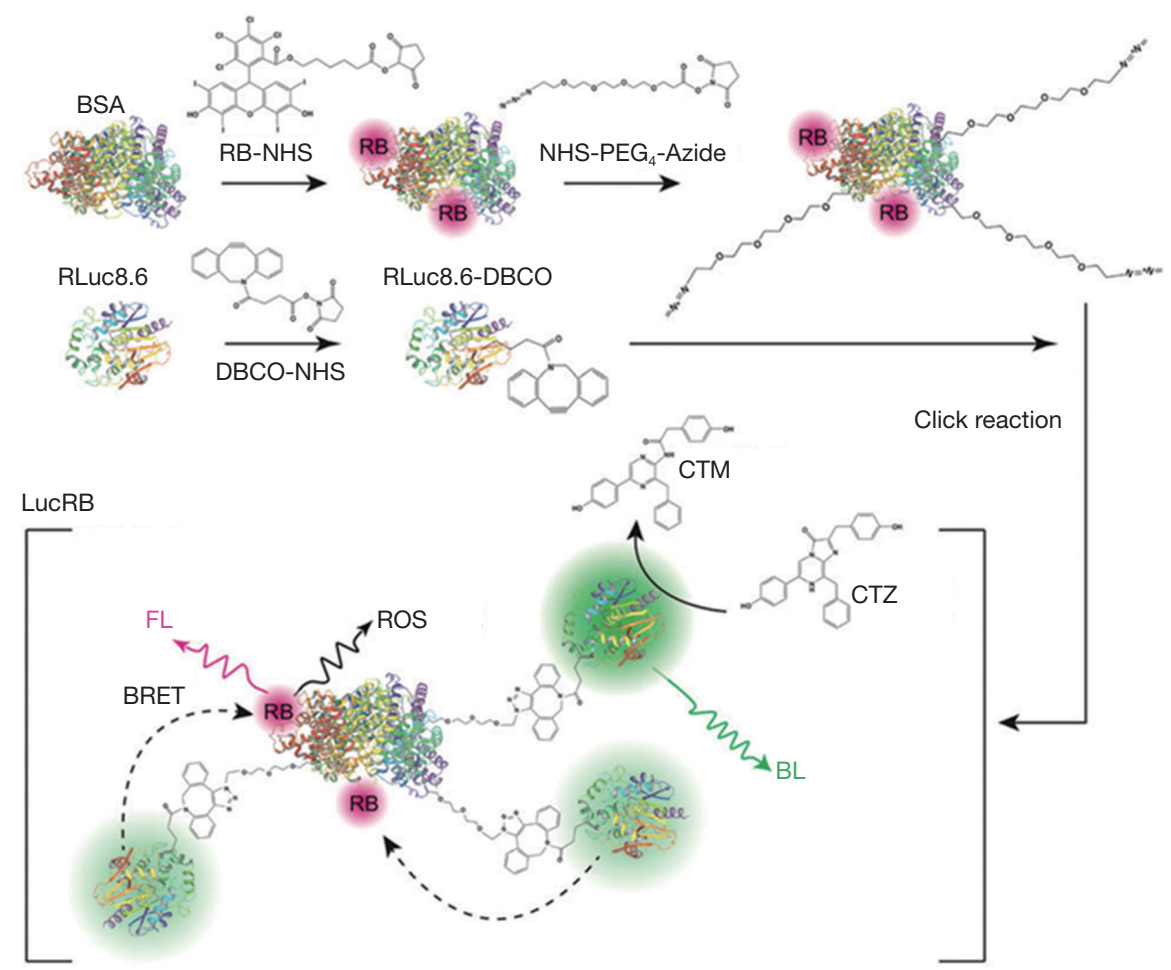

Figure 5 Bioluminescence-based photodynamic therapy with rose bengal-luciferase conjugates. Reproduced with permission from (38). ROS, reactive oxygen species; CTM, coelenteramide; CTZ, coelenterazine; BRET, bioluminescence resonance energy transfer.

Therefore, we expect that the application area of NPs and PDT will be broader in future due to these trials and the significant improvement in clinical practice.

\section{Acknowledgements}

Funding: This research was supported by the Basic Science Research Program of the National Research Foundation of Korea (NRF) funded by the Ministry of Education (grant No. 2016R1C1B3013951).

\section{Footnote}

Conflicts of Interest: The authors have no conflicts of interest to declare.

\section{References}

1. Nishiyama N, Morimoto Y, Jang WD, Kataoka K. Design and development of dendrimer photosensitizer-incorporated polymeric micelles for enhanced photodynamic therapy. Adv Drug Deliv Rev 2009;61:327-38.
2. Celli JP, Spring BQ, Rizvi I, Evans CL, Samkoe KS, Verma S, Pogue BW, Hasan T. Imaging and Photodynamic Therapy: Mechanisms, Monitoring, and Optimization. Chem Rev 2010;110:2795-838.

3. Dolmans DE, Fukumura D, Jain RK. Photodynamic therapy for cancer. Nat Rev Cancer 2003;3:380-7.

4. Spring BQ, Rizvi I, Xu N, Hasan T. The role of photodynamic therapy in overcoming cancer drug resistance. Photochem Photobiol Sci 2015;14:1476-91.

5. Sun Y, Campisi J, Higano C, Beer TM, Porter P, Coleman I, True L, Nelson PS. Treatment-induced damage to the tumor microenvironment promotes prostate cancer therapy resistance through WNT16B. Nat Med 2012;18:1359.

6. Huang Z. A Review of Progress in Clinical Photodynamic Therapy. Technol Cancer Res Treat 2005;4:283-93.

7. Ryu JH, Koo H, Sun IC, Yuk SH, Choi K, Kim K, Kwon IC. Tumor-targeting multi-functional nanoparticles for theragnosis: New paradigm for cancer therapy. Adv Drug Deliv Rev 2012;64:1447-58.

8. Wáng YX, Idée JM. A comprehensive literatures update of clinical researches of superparamagnetic resonance 
iron oxide nanoparticles for magnetic resonance imaging. Quant Imaging Med Surg 2017;7:88-122.

9. Torchilin V. Tumor delivery of macromolecular drugs based on the EPR effect. Adv Drug Deliv Rev 2011;63:131-5.

10. Byrne JD, Betancourt T, Brannon-Peppas L. Active targeting schemes for nanoparticle systems in cancer therapeutics. Adv Drug Deliv Rev 2008;60:1615-26.

11. Zhao M, Dong L, Liu Z, Yang S, Wu W, Lin J. In vivo fluorescence imaging of hepatocellular carcinoma using a novel GPC3-specific aptamer probe. Quant Imaging Med Surg 2018;8:151-60.

12. Lee DE, Koo H, Sun IC, Ryu JH, Kim K, Kwon IC. Multifunctional nanoparticles for multimodal imaging and theragnosis. Chem Soc Rev 2012;41:2656-72.

13. van Nostrum CF. Polymeric micelles to deliver photosensitizers for photodynamic therapy. Adv Drug Deliv Rev 2004;56:9-16.

14. Choi J, Kim H, Choi Y. Theranostic nanoparticles for enzyme-activatable fluorescence imaging and photodynamic/chemo dual therapy of triple-negative breast cancer. Quant Imaging Med Surg 2015;5:656-64.

15. Son J, Yang SM, Yi G, Roh YJ, Park H, Park JM, Choi M-G, Koo H. Folate-modified PLGA nanoparticles for tumor-targeted delivery of pheophorbide a in vivo. Biochem Biophys Res Commun 2018;498:523-8.

16. Yoon HY, Koo H, Choi KY, Lee SJ, Kim K, Kwon IC, Leary JF, Park K, Yuk SH, Park JH, Choi K. Tumortargeting hyaluronic acid nanoparticles for photodynamic imaging and therapy. Biomaterials 2012;33:3980-9.

17. Luo Y, Wu H, Feng C, Xiao K, Yang X, Liu Q, Lin TY, Zhang H, Walton JH, Ajena Y, Hu Y, Lam KS, Li Y. "OnePot" Fabrication of Highly Versatile and Biocompatible Poly(vinyl alcohol)-porphyrin-based Nanotheranostics. Theranostics 2017;7:3901-14.

18. Spring BQ, Bryan Sears R, Zheng LZ, Mai Z, Watanabe R, Sherwood ME, Schoenfeld DA, Pogue BW, Pereira SP, Villa E, Hasan T. A photoactivable multi-inhibior nanoliposome for tumour control and simultaneous inhibition of treatment escape pathways. Nat Nanotechnol 2016;11:378-87.

19. Wang Y, Xie Y, Li J, Peng ZH, Sheinin Y, Zhou J, Oupický D. Tumor-Penetrating Nanoparticles for Enhanced Anticancer Activity of Combined Photodynamic and Hypoxia-Activated Therapy. ACS Nano 2017;11:2227-38.

20. Wang J, Zhong Y, Wang X, Yang W, Bai F, Zhang B, Alarid L, Bian K, Fan H. pH-Dependent Assembly of PorphyrinSilica Nanocomposites and Their Application in Targeted
Photodynamic Therapy. Nano Lett 2017;17:6916-21.

21. Idris NM, Gnanasammandhan MK, Zhang J, Ho PC, Mahendran R, Zhang Y. In vivo photodynamic therapy using upconversion nanoparticles as remote-controlled nanotransducers. Nat Med 2012;18:1580-5.

22. Ge J, Lan M, Zhou B, Liu W, Guo L, Wang H, Jia Q, Niu G, Huang X, Zhou H, Meng X, Wang P, Lee CS, Zhang W, Han X. A graphene quantum dot photodynamic therapy agent with high singlet oxygen generation. Nat Commun 2014;5:4596.

23. Mei J, Leung NL, Kwok RT, Lam JW, Tang BZ. Aggregation-Induced Emission: Together We Shine, United We Soar! Chem Rev 2015;115:11718-940.

24. Hong SH, Kim H, Choi Y. Indocyanine green-loaded hollow mesoporous silica nanoparticles as an activatable theranostic agent. Nanotechnology 2017;28:185102.

25. Hong SH, Kim H, Choi Y. Enhanced Fluorescence Imaging and Photodynamic Cancer Therapy Using Hollow Mesoporous Nanocontainers. Chem Asian J 2017;12:1700-3.

26. Yuan Y, Feng G, Qin W, Tang BZ, Liu B. Targeted and image-guided photodynamic cancer therapy based on organic nanoparticles with aggregation-induced emission characteristics. Chem Commun (Camb) 2014;50:8757-60.

27. Li M, Gao Y, Yuan Y, Wu Y, Song Z, Tang BZ, Liu B, Zheng QC. One-Step Formulation of Targeted Aggregation-Induced Emission Dots for Image-Guided Photodynamic Therapy of Cholangiocarcinoma. ACS Nano 2017;11:3922-32.

28. Gu B, Wu W, Xu G, Feng G, Yin F, Chong PH, Qu J, Yong KT, Liu B. Precise Two-Photon Photodynamic Therapy using an Efficient Photosensitizer with Aggregation-Induced Emission Characteristics. Adv Mater 2017;29(28).

29. Jayaram DT, Ramos-Romero S, Shankar BH, Garrido C, Rubio N, Sanchez-Cid L, Gómez SB, Blanco J, Ramaiah D. In Vitro and in Vivo Demonstration of Photodynamic Activity and Cytoplasm Imaging through TPE Nanoparticles. ACS Chem Biol 2016;11:104-12.

30. Chang CC, Hsieh MC, Lin JC, Chang TC. Selective photodynamic therapy based on aggregationinduced emission enhancement of fluorescent organic nanoparticles. Biomaterials 2012;33:897-906.

31. Yuan Y, Zhang CJ, Gao M, Zhang R, Tang BZ, Liu B. Specific Light-Up Bioprobe with Aggregation - Induced Emission and Activatable Photoactivity for the Targeted and Image - Guided Photodynamic Ablation of Cancer Cells. Angew Chem Int Ed Engl 2015;54:1780-6. 
32. Feng G, Qin W, Hu Q, Tang BZ, Liu B. Cellular and Mitochondrial Dual-Targeted Organic Dots with Aggregation-Induced Emission Characteristics for ImageGuided Photodynamic Therapy. Adv Healthc Mater 2015;4:2667-76.

33. Han K, Wang SB, Lei Q, Zhu JY, Zhang XZ. Ratiometric Biosensor for Aggregation-Induced EmissionGuided Precise Photodynamic Therapy. ACS Nano 2015;9:10268-77.

34. Guan Y, Lu H, Li W, Zheng Y, Jiang Z, Zou J, Gao H. Near-Infrared Triggered Upconversion Polymeric Nanoparticles Based on Aggregation-Induced Emission and Mitochondria Targeting for Photodynamic Cancer Therapy. ACS Appl Mater Interfaces 2017;9:26731-9.

35. Yuan H, Chong H, Wang B, Zhu C, Liu L, Yang Q, Lv F, Wang S. Chemical Molecule-Induced Light-Activated System for Anticancer and Antifungal Activities. J Am

Cite this article as: Yi G, Hong SH, Son J, Yoo J, Park C, Choi Y, Koo H. Recent advances in nanoparticle carriers for photodynamic therapy. Quant Imaging Med Surg 2018;8(4):433443. doi: 10.21037/qims.2018.05.04
Chem Soc 2012;134:13184-7.

36. Mao D, Wu W, Ji S, Chen C, Hu F, Kong D, Ding D, Liu B. Chemiluminescence-Guided Cancer Therapy Using a Chemiexcited Photosensitizer. Chem 2017;3:991-1007.

37. Hsu CY, Chen CW, Yu HP, et al. Bioluminescence resonance energy transfer using luciferase-immobilized quantum dots for self-illuminated photodynamic therapy. Biomaterials 2013;34:1204-12.

38. Kim YR, Kim S, Choi JW, Choi SY, Lee SH, Kim H, Hahn SK, Koh GY, Yun SH. Bioluminescence-activated deep-tissue photodynamic therapy of cancer. Theranostics 2015;5:805-17.

39. Kim S, Jo H, Jeon M, Choi MG, Hahn SK, Yun SH. Luciferase-Rose Bengal conjugates for singlet oxygen generation by bioluminescence resonance energy transfer. Chem Commun (Camb) 2017;53:4569-72. 